\title{
Health Related Quality of Life of Patients Undergoing In-Centre Hemodialysis in Rwanda: a Cross Sectional Study
}

\author{
Gloria SHUMBUSHO ( $\sim$ glorishu@gmail.com ) \\ University of Rwanda \\ Celestin HATEGEKA \\ Harvard TH Chan School of Public Health, Boston MA USA \\ Marianne Vidler \\ University of British Columbia \\ Jules KABAHIZI \\ Rwanda Military Hospital, University of Rwanda \\ Marla MCKNIGHT \\ University of British, Harvard Medical School
}

\section{Research Article}

Keywords: Health related quality of life, patient reported outcomes measures, end stage kidney disease, in-centre hemodialysis, Rwanda

Posted Date: March 1st, 2022

DOI: https://doi.org/10.21203/rs.3.rs-1407207/v1

License: (c) (1) This work is licensed under a Creative Commons Attribution 4.0 International License. Read Full License 


\section{Abstract \\ Background}

There are few studies assessing the quality of life of patients with chronic and end stage kidney disease in sub-Saharan Africa. We aimed to describe the health-related quality of life (HRQOL) of patients undergoing in-centre maintenance hemodialysis in Rwanda using the $\mathrm{KDQOL}^{\mathrm{TM}}-36$ and determine sociodemographic and clinical factors associated with their quality of life.

\section{Methods}

We conducted a multicenter, cross-sectional study between September 2020 and March 2021. Patients over the age of 18 receiving maintenance in-centre hemodialysis for at least 3 months at the Rwandan tertiary hospitals were administered the $\mathrm{KDQOL}^{\mathrm{TM}}-36$ questionnaire to assess physical and mental health functioning, the effect, burden and symptoms and problem of kidney disease. Sociodemographic and clinical information were also collected for all eligible patients. Using mixed effects linear regression models, we explored factors associated with overall KDQOL and its domains, while accounting for clustering of patients within hemodialysis centres.

\section{Results}

Eighty-nine eligible patients were included in the study. The majority of participants were younger than 60 years old (69.7\%), male (66.3\%), had comorbidities (91\%), and $71.6 \%$ were categorized as level 3 on a 4 tier in-country poverty scale. All participants had health insurance coverage, with $67.4 \%$ bearing no out of pocket payments for hemodialysis. The mean (SD) quality of life score was 48.92 (18.84) for overall HRQOL, 37.33 (10.66) for PCS and 44.74 (9.98) for MCS. Symptoms and problem of kidney disease, effect of kidney disease, and burden of kidney disease scored 58.22 (27.44), 53.48 (17.14) and 20.01 (18.27), respectively. A notable difference of KDQOL scores between hemodialysis centres was observed. Overall KDQOL was associated with male sex (aß [adjusted ß coefficient]: 8.54, 95\% confidence interval [Cl]: 2.77, 14.26); being employed (aß: 8.16, 95\% Cl: 2.18, 14.29); dialysis vintage of 13-24 months (aß: 10.47, 95\% Cl: 3.57, 17.58), hemoglobin of $10-11 \mathrm{~g} / \mathrm{dl}(\mathrm{a}: 7.27,95 \% \mathrm{Cl}: 0.70$, 13.72) and comorbidities (e.g., 4 comorbidities vs none (aß: -29.76, 95\% Cl: -41.47, -18.32).

\section{Conclusions}

Patients on in-centre hemodialysis in Rwanda have reduced KDQOL scores, particularly in the burden of kidney disease and physical composite summary domains. Higher overall KDQOL mean score was associated with male sex, being employed, and dialysis vintage of 13-24 months, hemoglobin of $10-11 \mathrm{~g} / \mathrm{dl}$ and absence of comorbidities. Majority of patients have higher socioeconomic status reflecting the social and financial constraints to access and maintain dialysis in resource limited settings.

\section{Background}

The burden of kidney disease worldwide is substantial, growing and poses significant challenges for governments responding to the health of their populations, particularly in low and middle income countries (LMIC).(1) In middle and eastern Africa, access to renal replacement therapy (RRT) is estimated at 1-3\% (7), and the outcome of dialysis patients is poor, and marked by premature mortality in the first year after dialysis initiation.(8)(9) A high mortality rate following initiation of dialysis may be related to late presentation to a nephrologist or kidney care center, affordability, lack of access to treatment for metabolic complications and poor education.(9)

Rwanda is one of the smallest central African countries with an approximately 13 million population and only about $17.6 \%$ living in urban areas.(10) The gross domestic product per capita is approximately 820 US dollars.(11) Over $92 \%$ of Rwandans access health care using community based health insurance ( $\mathrm{CBHI}$ ) linked to four socioeconomic life standing of Rwandan households, also named "Ubudehe categories".(12)(13) Patients in category 1, are exempt from premiums; category 2,3 and 4 pay a fixed co- 
pay for health center and hospital visits.(14) Approximately $6 \%$ of the total population have civil servant health insurance and military medical insurance (MMI).(12)(13)(14) In addition, there are private health insurance schemes available for purchase, and funds that cover medical care for vulnerable groups, such as genocide victim funds (GVF).

Based on estimates from World Health Organization, noncommunicable diseases (NCDs) including renal diseases were predominant cause of mortality in Rwanda, accounting for $58 \%$ of the mortality burden since 2016.(15) There are insufficient data on the prevalence of kidney diseases in Rwanda but kidney failure was among the top 10 leading cause of death from noncommunicable diseases and injuries in Rwanda in 2016.(15)(16) Hemodialysis is the predominant renal replacement therapy available in Rwanda, very few patients are currently on peritoneal dialysis and renal transplantation is not performed in the country. Patients access transplantation through the Ministry of Health funded transplantation performed out of country or through out-of-pocket payments abroad.(16)

Access to dialysis is limited by its cost, a shortage of specialized medical staff with training in nephrology and renal replacement therapy and geographic distribution of in-centre hemodialysis units.(16)(17) The annual cost of hemodialysis per patient in Rwanda ranges between Int\$13,260 and Int\$20,592. CBHI covers hemodialysis for only six weeks for patients with acute kidney injury (AKI) and does not cover costs associated with chronic RRT for CKD.(18) Maintenance dialysis is covered by employer and private health insurances or special funds (eg GVF) at 85 to $100 \%$ of HD cost, thus, for the majority of Rwandans, there are substantial out of pocket costs and financial hardship associated with hemodialysis.(16)

Same as other specialized care centered in urban areas, dialysis centers are located in cities, particularly Kigali City; however, the majority of Rwandans live in rural areas.(10). Currently, in-centre maintenance hemodialysis is available at three public, university affiliated tertiary referral centers, Kigali University Teaching Hospital (CHUK), Rwanda Military hospital (RMH) located in the capital city, Kigali and Butare University Teaching Hospital (CHUB) built in the south province, and at King Faisal Hospital (KFH), which is a public-private quaternary hospital also located in Kigali. Community based hemodialysis is provided by African Health Network, a private company with three unities located at Kimihurura (Kigali), Rubavu and Rusizi (Western province) with relatively similar cost, insurance coverage and out of pocket expenses as in-centre hemodialysis.(16)

Between 2014 and 2017, approximately 47\% of hemodialysis patients died within four months of initiation of dialysis at CHUK. (18)(19) There are dialysis dependent and non-dialysis dependent factors that impact patient experience and outcomes beyond dialysis adequacy including socioeconomic status, age, comorbidities, vascular access, dialysis session duration and adequacy, dialysis session frequency, and symptoms associated with dialysis.(20)(21)(22)(23)(24)(25)(26)

To promote high-quality services in renal dialysis facilities, routine measurement of patient reported outcomes measures such health related quality of life (HRQOL) in dialysis patients using the KDQOL-36 ${ }^{\mathrm{Tm}}$ is now mandatory in high income countries. For example, In the United States, these measurements are typically done four months after initiation of dialysis and at least every year.(27)(28) The Kidney Disease Quality of Life (KDQOL) instrument designed by RAND Health Care and validated by the National Kidney Foundation for patients with kidney diseases may provide a reasonable metric of quality of life of adult patients living with ESKD in resource limited settings.(29)(30)(31) In Africa, there are few studies that assessed the QOL of patients with CKD, less again of dialysis patients. Masina et al. (32) in Malawi and Kamau et al. (33) in Kenya reported reduced HRQOL of dialysis patients.

In this study, we aim to determine the health related quality of life of patients with ESKD undergoing in-centre maintenance hemodialysis in Rwanda, describe demographic and clinical features of those patients and the factors associated with their quality of life. This is the first study in Rwanda and will provide baseline data that can help inform improvement strategies for ESKD patients on hemodialysis in Rwanda and other settings with similar contexts.

\section{Methods}

We conducted a multicenter prospective cross-sectional study on all patients with end-stage kidney disease on in-centre hemodialysis in Rwanda between September 2020 and March 2021. In-centre hemodialysis units are located at Kigali University Teaching Hospital (CHUK), Rwanda Military hospital (RMH), Butare University Teaching Hospital (CHUB) and at King Faisal

Page $3 / 16$ 
Hospital (KFH). We enrolled 89 adults patients (18 years and above) with ESKD on in-centre maintenance hemodialysis for at least three months (Fig. 1). We excluded patients with AKI, hospitalized within the last four weeks and/or with neurological disability making them unable to respond to the questions.

\section{Data collection and measures}

HRQOL data were collected using the KDQOL36-Item Short Form questionnaire. Instructions and documentations on scoring were obtained from RAND Health Care.(29) Because of relatively small number of participants at CHUK and CHUB, results from these two in-centre hemodialysis units were aggregated for analysis. To determine factors associated with HRQOL of hemodialysis patients, sociodemographic and clinical data were collected and all cutoffs were based on distribution of the data. We collected data regarding hemodialysis. We did not collect data about small solutes clearance using Kt/V as it was not measured in most dialysis centers. A questionnaire was administered to each participant during their regularly scheduled dialysis in person by one study investigator to ensure clarifying questions that can be asked by the participant. For patients not fluent in English, questions were translated by the study team into local language before administration. After obtaining informed consent, participants anonymously and privately completed the questionnaire in person or with help of an investigator. Approximately 10 minutes were required for each patient to fill the survey.

Responses on quality of life questionnaire were exported to excel scoring tool of KDQOL-36 ${ }^{\text {T"' }}$ which provided patients' score in five domains ( Physical composite summary PCS, Mental composite summary MCS, Burden of kidney disease BKD, Effect of kidney disease EKD and Symptoms and problem of kidney disease SPKD). The overall KDQOL score was obtained from a programed KDQOL-36 $6^{\text {Tw }}$ survey.(34) All demographic and clinical information were collected on paper, entered into excel with independent double entry by two study investigators to minimize data entry errors.

\section{Analysis}

Descriptive statistics were used to describe the study sample including demographic and clinical characteristics overall and by KDQOL. Mean and standard deviation (or median with inter-quartile range) were used, as appropriate, for continuous variables and frequency (and percentage) for categorical variables. Mixed effects linear regression models were fitted to explore factors associated with overall KDQOL and its five domains, while accounting for clustering of patients within hemodialysis centers. Parameter estimates are reported as $\beta$ coefficients along with their $95 \%$ confidence interval $(\mathrm{Cl})$ and $p$ values. All analyses were conducted using $\mathrm{R}$ version 4.0.2.

\section{Results}

\section{Demographic and clinical characteristics of study participants}

Majority of participants were young with male to female ratio of nearly 2:1. Almost all participants (91\%) had comorbidities and $79.8 \%$ were taking > 3 medications per day. More than half (59.5\%) have been on hemodialysis for more than 12 months with $80.9 \%$ on thrice weekly hemodialysis (Table 1 ).

\section{Health related quality of life}

The overall mean \pm SD quality of life score was $48.92 \pm 18.84$. The mean physical composite summary (PCS) score was $37.33 \pm$ 10.66 and mental composite summary (MCS) mean score was $44.74 \pm 9.98$. Symptoms and problems of kidney disease (SPKD), effect of kidney disease (EKD), and burden of kidney disease (BKD) had respectively a mean (SD) scores of 58.22 (27.44), 53.48 (17.14) and 20.01 (18.27) (Figure 2). Comparison between hemodialysis centers showed significant difference of HRQOL scores between hemodialysis centers and the overall KDQOL score and SPKD, BKD, PCS and MCS (P value <0.001) and King Faisal Hospital hemodialysis unit had the highest HRQOL mean scores in all domains.

KDQOL varied by independent variables. Significantly higher mean quality of life scores were observed in patients 45-60 years (53.39 \pm 17.67$)$, with a serum albumin level above $40 \mathrm{~g} / \mathrm{dl}(52.39 \pm 16.96)$, on hemodialysis for $13-24$ months $(56.63 \pm 15.31)$ ), with 
a fistula for hemodialysis access $(58.40 \pm 19.77)$ and without comorbidities $(68.80 \pm 13.32)$.

Table 1. Demographic and clinical characteristics of study participants 


\begin{tabular}{|c|c|c|c|}
\hline \multirow[b]{2}{*}{ Variable } & \multirow[b]{2}{*}{ Category } & \multicolumn{2}{|l|}{ Sample } \\
\hline & & $\mathrm{n}=89$ & $\%$ \\
\hline \multirow[t]{2}{*}{ Sex } & Female & 30 & 33.7 \\
\hline & Male & 59 & 66.3 \\
\hline \multirow[t]{3}{*}{ Age } & $<45$ years & 29 & 32.6 \\
\hline & $45-60$ years & 33 & 37.1 \\
\hline & $>60$ years & 27 & 30.3 \\
\hline \multirow[t]{3}{*}{ Education } & Post-secondary & 36 & 40.4 \\
\hline & Secondary school & 26 & 29.2 \\
\hline & Primary or less & 27 & 30.3 \\
\hline \multirow[t]{3}{*}{ Marital status } & Married/ living together & 48 & 53.9 \\
\hline & Never married & 23 & 25.8 \\
\hline & Separated or widowed & 18 & 20.2 \\
\hline \multirow[t]{4}{*}{ Ubudehe category } & 1 & 7 & 8.0 \\
\hline & 2 & 18 & 20.5 \\
\hline & 3 & 63 & 71.6 \\
\hline & 4 & 0 & 0 \\
\hline \multirow[t]{2}{*}{ Insurance coverage } & $<100 \%$ coverage & 29 & 32.6 \\
\hline & $100 \%$ coverage & 60 & 67.4 \\
\hline \multirow[t]{3}{*}{ Hemodialysis centers } & CHUK-CHUB & 14 & 15.7 \\
\hline & King Faisal Hospital & 43 & 48.3 \\
\hline & Rwanda Military Hospital & 32 & 36.0 \\
\hline \multirow[t]{2}{*}{ Employment status } & Employed & 27 & 30.3 \\
\hline & Retired & 12 & 13.5 \\
\hline \multirow[t]{4}{*}{ Number of comorbidities } & 0 & 8 & 9.0 \\
\hline & 1 & 44 & 49.4 \\
\hline & 2 & 28 & 31.5 \\
\hline & 3 & 9 & 10.1 \\
\hline \multirow[t]{3}{*}{ Number of medications } & $<3$ drugs & 18 & 20.2 \\
\hline & 3-4 drugs & 38 & 42.7 \\
\hline & $>4$ drugs & 33 & 37.1 \\
\hline \multirow[t]{2}{*}{ Hospitalized in the last 6 months $(n=87)$} & No & 40 & 46.0 \\
\hline & Yes & 47 & 54.0 \\
\hline \multirow[t]{3}{*}{ Albumin g/l } & $<35$ & 21 & 23.6 \\
\hline & $35-40$ & 40 & 44.9 \\
\hline & $>40$ & 28 & 31.5 \\
\hline \multirow[t]{3}{*}{ Hemoglobin g/dl } & $<10$ & 38 & 42.7 \\
\hline & $10-11$ & 24 & 27.0 \\
\hline & $>11$ & 27 & 30.3 \\
\hline \multirow[t]{2}{*}{ Number of HD per week } & 2 & 17 & 19.1 \\
\hline & 3 & 72 & 80.9 \\
\hline \multirow[t]{2}{*}{ Number of HD in past 30 days } & $\leq 10$ sessions & 28 & 31.5 \\
\hline & $>10$ sessions & 61 & 68.5 \\
\hline \multirow[t]{3}{*}{ Hemodialysis access } & Fistula/graft & 30 & 33.7 \\
\hline & Semi-permanent dialysis catheter & 34 & 38.2 \\
\hline & Temporary dialysis catheter & 25 & 28.1 \\
\hline \multirow[t]{4}{*}{ Dialysis vintage } & $<12$ months & 36 & 40.4 \\
\hline & 13-24 months & 22 & 24.7 \\
\hline & $>24$ months & 31 & 34.8 \\
\hline & Unemployed & 50 & 56.2 \\
\hline
\end{tabular}

\#Ubudehe category: economic life standing of households of Rwandan population 


\section{Factors associated with health related quality of life}

In adjusted model; sex, employment status, dialysis vintage, number of comorbidities, and hemoglobin level correlated significantly with overall kidney disease quality of life. Sex, employment status, type of hemodialysis access and comorbidities were statistically significantly associated with physical composite summary domain. Employment status, dialysis vintage and number of comorbidities were significantly associated with mental composite summary domain (Table 2). The burden of kidney disease subscale correlated significantly with employment status, type of hemodialysis access and number of comorbidities. Number of hemodialysis in the past 30 days and number of comorbidities were significantly associated with the effect of kidney disease. Age, sex, dialysis vintage and number of comorbidities were significantly associated with symptoms and problems of kidney disease subscale (Table 3).

\section{Discussion}

In the current study, we found reduced quality of life mean scores. Our results were relatively lower or similar to studies from middle and higher income countries that have used the same KDQOL-36 $6^{\text {TM }}$ tool and others from SSA, though they used the KDQOL-SF 1.3 version. For example, Masina et al in Malawi (32) and Bagasha et al in Uganda (35) found an overall HRQOL score of $59.9( \pm 8.8)$ and $41.71( \pm 4.42)$ respectively (Table 4). The BKD had a lower score $(20.01 \pm 18.27)$ and Symptoms and problem of kidney disease domain had relatively higher mean score $(58.22 \pm 27.44)$ more marked in elder patients above 60 ( $13.611 ; 95 \% \mathrm{Cl}: 1.42,26.29)$. Similar findings of low scores in the burden of kidney disease sub-scale and relatively higher score in the symptoms and problem of kidney disease sub-scale have been noted in studies conducted Africa.(32)(33)(35) Similar to other studies from LMICs, $69.7 \%$ of participants were young than 60 reflecting the pattern of causes and risk factors of kidney disease in LMICs, the lack of strategies of prevention and management of communicable and non-communicable diseases and the poor socioeconomic status and the low access to transplantation.(36)(16)(33)(35)(32)(37)(38)(25) In our study, the majority of patients were living in Kigali where three of four in-centre hemodialysis units are located. Therefore patients and their family have to travel long distances or move to near dialysis centers affecting their daily activities, and relationship with others. Our findings showed that the majority of patients on hemodialysis in Rwanda were largely from a relatively higher socioeconomic status, echoing other African studies.(32)(33)(35)(38)(39)(16) Among our study participants, 53.9\% were married living together with their partner, $68.5 \%$ were living in urban area and $70.7 \%$ had secondary school level or post-secondary. Despite that a small proportion of Rwandan population is categorized in "ubudehe" category three and four (13), $71.6 \%$ of our study participants were classified in third category. This echoes findings from other African studies that have highlighted that the majority of patients on hemodialysis are largely from a relatively higher socioeconomic status.(32)(33)(35)(38)(39)(16). Similarly, all participants had health insurance coverage, with $67.4 \%$ covered at $100 \%$. These findings reflect the financial constraints and the social impact to access and maintain RRT in resource limited settings.

Table 2. Factors associated with overall KDQOL, PCS and MCS 


\begin{tabular}{|c|c|c|c|c|c|c|c|c|c|c|c|c|}
\hline \multirow{2}{*}{$\begin{array}{l}\text { ndependent variables } \\
\text { (reference group) }\end{array}$} & \multicolumn{2}{|c|}{ Overall KDQOL } & \multirow[b]{2}{*}{$\begin{array}{l}\text { Adjusted B } \\
\text { coefficient } \\
(95 \% \mathrm{CI})\end{array}$} & \multirow[b]{2}{*}{$\begin{array}{l}P \\
\text { value }\end{array}$} & \multicolumn{4}{|c|}{ Physical.Composite Summary } & \multicolumn{4}{|c|}{ Mental.Composite Summary } \\
\hline & $\begin{array}{l}\text { Crude } B \\
\text { coefficient } \\
(95 \% \mathrm{CI})\end{array}$ & $\begin{array}{l}\mathrm{P} \\
\text { value }\end{array}$ & & & $\begin{array}{l}\text { Crude B } \\
\text { coefficient } \\
(95 \% \mathrm{CI})\end{array}$ & $\begin{array}{l}\mathrm{P} \\
\text { value }\end{array}$ & $\begin{array}{l}\text { Adjusted B } \\
\text { coefficient } \\
(95 \% \mathrm{CI})\end{array}$ & $\begin{array}{l}P \\
\text { value }\end{array}$ & $\begin{array}{l}\text { Crude } B \\
\text { coefficient } \\
(95 \% \mathrm{CI})\end{array}$ & $\begin{array}{l}\mathrm{P} \\
\text { value }\end{array}$ & $\begin{array}{l}\text { Adjusted B } \\
\text { coefficient } \\
(95 \% \mathrm{CI})\end{array}$ & $\begin{array}{l}P \\
\text { value }\end{array}$ \\
\hline \multicolumn{13}{|l|}{ Sex (Female) } \\
\hline Male & $\begin{array}{l}6.57(0.02 \\
13.04)\end{array}$ & 0.04 & $\begin{array}{l}8.54(2.77 \\
14.26)\end{array}$ & 0.008 & $\begin{array}{l}6.84(3.24 \\
10.41)\end{array}$ & $<0.001$ & $\begin{array}{l}4.62(1.02 \\
8.21)\end{array}$ & 0.01 & $\begin{array}{l}-0.45 \\
(-4.39 \\
3.42)\end{array}$ & 0.81 & $\begin{array}{l}0.61 \\
(-2.86 \\
4.12)\end{array}$ & 0.74 \\
\hline \multicolumn{13}{|l|}{$\operatorname{tge}(<45$ years $)$} \\
\hline $5-60$ years & $\begin{array}{l}3.35(-4.32 \\
11.49)\end{array}$ & 0.40 & $\begin{array}{l}6.23 \\
(-0.37 \\
13.14)\end{array}$ & 0.09 & $\begin{array}{l}-2.76 \\
(-7.19 \\
1.91)\end{array}$ & 0.23 & $\begin{array}{l}-1.32 \\
(-5.40 \\
2.96)\end{array}$ & 0.55 & $\begin{array}{l}4.04 \\
(-0.37 \\
8.85)\end{array}$ & 0.08 & $\begin{array}{l}3.83 \\
(-0.17 \\
8.39)\end{array}$ & 0.08 \\
\hline$>60$ years & $\begin{array}{l}5.37(-2.43 \\
13.47)\end{array}$ & 0.18 & $\begin{array}{l}6.68 \\
(-1.67 \\
15.17)\end{array}$ & 0.15 & $\begin{array}{l}-3.92 \\
(-8.42 \\
0.74)\end{array}$ & 0.09 & $\begin{array}{l}-4.19 \\
(-9.50 \\
1.20)\end{array}$ & 0.14 & $\begin{array}{l}4.56(0.05 \\
9.31)\end{array}$ & 0.05 & $\begin{array}{l}1.09 \\
(-3.86, \\
6.36)\end{array}$ & 0.68 \\
\hline \multicolumn{13}{|c|}{ Żducation (Primary school and less) } \\
\hline Secondary school & $\begin{array}{l}2.38(-6.06 \\
10.56)\end{array}$ & 0.56 & & & $\begin{array}{l}0.75 \\
(-4.16 \\
5.52)\end{array}$ & 0.75 & & & $\begin{array}{l}2.58 \\
(-2.24 \\
7.45)\end{array}$ & 0.24 & & \\
\hline ?ost-secondary & $\begin{array}{l}2.17(-5.33 \\
9.57)\end{array}$ & 0.57 & & & $\begin{array}{l}0.62 \\
(-3.76 \\
4.93)\end{array}$ & 0.77 & & & $\begin{array}{l}2.70 \\
(-1.81 \\
6.89)\end{array}$ & 0.27 & & \\
\hline \multicolumn{13}{|c|}{ Marital status (Never married) } \\
\hline $\begin{array}{l}\text { Married/living } \\
\text { ogether }\end{array}$ & $\begin{array}{l}3.59(-3.63 \\
10.89)\end{array}$ & 0.33 & & & $\begin{array}{l}-1.09 \\
(-5.17 \\
3.01)\end{array}$ & 0.60 & & & $\begin{array}{l}1.37 \\
(-2.94 \\
5.75)\end{array}$ & 0.53 & & \\
\hline ¡eparated/widowed & $\begin{array}{l}-4.77(-13.75 \\
4.31)\end{array}$ & 0.30 & & & $\begin{array}{l}-7.61 \\
(-12.67 \\
-2.49)\end{array}$ & 0.004 & & & $\begin{array}{l}-1.48 \\
(-6.84 \\
3.96)\end{array}$ & 0.59 & & \\
\hline \multicolumn{13}{|c|}{ Imployment (Unemployed) } \\
\hline Imployed & $\begin{array}{l}5.99(-0.85 \\
12.89)\end{array}$ & 0.09 & $\begin{array}{l}8.16(2.18 \\
14.29)\end{array}$ & 0.01 & $\begin{array}{l}4.85(0.92 \\
8.80)\end{array}$ & 0.01 & $\begin{array}{l}3.88(0.39 \\
7.39)\end{array}$ & 0.04 & $\begin{array}{l}2.32 \\
(-1.66 \\
6.34)\end{array}$ & 0.25 & $\begin{array}{l}5.76 \\
(2.09 \\
9.53)\end{array}$ & 0.005 \\
\hline Retired & $\begin{array}{l}6.93(-2.32 \\
16.37)\end{array}$ & 0.14 & $\begin{array}{l}4.23 \\
(-5.01 \\
13.70)\end{array}$ & 0.41 & $\begin{array}{l}4.18 \\
(-1.12 \\
9.58)\end{array}$ & 0.12 & $\begin{array}{l}5.68(0.05 \\
11.40)\end{array}$ & 0.06 & $\begin{array}{l}6.45(1.07 \\
11.96)\end{array}$ & 0.02 & $\begin{array}{l}8.18 \\
(2.66, \\
13.86)\end{array}$ & 0.007 \\
\hline \multicolumn{13}{|c|}{ Jbudehe category (1) } \\
\hline 2 & $\begin{array}{l}1.78(-10.99 \\
14.66)\end{array}$ & 0.78 & & & $\begin{array}{l}-0.75 \\
(-7.75 \\
6.30)\end{array}$ & 0.83 & & & $\begin{array}{l}-2.87 \\
(-10.46 \\
4.80)\end{array}$ & 0.46 & & \\
\hline 3 & $\begin{array}{l}6.84(-4.62 \\
18.56)\end{array}$ & 0.25 & & & $\begin{array}{l}4.41 \\
(-1.87 \\
10.83)\end{array}$ & 0.17 & & & $\begin{array}{l}-1.04 \\
(-7.84 \\
5.96)\end{array}$ & 0.76 & & \\
\hline \multicolumn{13}{|c|}{$\begin{array}{l}\text { Health insurance coverage }(<100 \% \\
\text { :overage) }\end{array}$} \\
\hline 00\% coverage & $\begin{array}{l}4.77(-1.84 \\
11.28)\end{array}$ & 0.15 & & & $\begin{array}{l}0.34 \\
(-3.55 \\
4.17)\end{array}$ & 0.86 & & & $\begin{array}{l}1.08 \\
(-2.86 \\
4.93)\end{array}$ & 0.58 & & \\
\hline \multicolumn{13}{|c|}{ Number of HD per week (Twice) } \\
\hline Thrice & $\begin{array}{l}-1.47(-9.67 \\
6.46)\end{array}$ & 0.71 & & & $\begin{array}{l}-0.48 \\
(-5.26 \\
4.13)\end{array}$ & 0.83 & & & $\begin{array}{l}-0.30 \\
(-5.15 \\
4.35)\end{array}$ & 0.89 & & \\
\hline \multicolumn{13}{|c|}{$\begin{array}{l}\text { Number of HD in the past } 30 \text { days ( } \leq \\
\text { l0) }\end{array}$} \\
\hline$>10$ & $\begin{array}{l}-2.53(-9.19 \\
4.09)\end{array}$ & 0.45 & & & $\begin{array}{l}-0.39 \\
(-4.27 \\
3.47)\end{array}$ & 0.84 & & & $\begin{array}{l}-0.15 \\
(-4.08 \\
3.74)\end{array}$ & 0.93 & & \\
\hline \multicolumn{13}{|c|}{ Tospitalized in the last 6 months (No) } \\
\hline les & $\begin{array}{l}-3.61(-9.88 \\
2.63)\end{array}$ & 0.25 & & & $\begin{array}{l}-4.68 \\
(-8.22 \\
-1.15)\end{array}$ & 0.01 & & & $\begin{array}{l}-1.97 \\
(-5.66 \\
1.68)\end{array}$ & 0.29 & & \\
\hline
\end{tabular}




\begin{tabular}{|c|c|c|c|c|c|c|c|c|c|c|c|c|}
\hline \multirow{2}{*}{$\begin{array}{l}\text { ndependent variables } \\
\text { reference group) }\end{array}$} & \multicolumn{2}{|c|}{ Overall KDQOL } & \multirow[b]{2}{*}{$\begin{array}{l}\text { Adjusted B } \\
\text { coefficient } \\
(95 \% \mathrm{CI})\end{array}$} & \multirow[b]{2}{*}{$\begin{array}{l}P \\
\text { value }\end{array}$} & \multicolumn{4}{|c|}{ Physical.Composite Summary } & \multicolumn{4}{|c|}{ Mental.Composite Summary } \\
\hline & $\begin{array}{l}\text { Crude B } \\
\text { coefficient } \\
(95 \% \mathrm{CI})\end{array}$ & $\begin{array}{l}P \\
\text { value }\end{array}$ & & & $\begin{array}{l}\text { Crude B } \\
\text { coefficient } \\
(95 \% \mathrm{CI})\end{array}$ & $\begin{array}{l}\mathrm{P} \\
\text { value }\end{array}$ & $\begin{array}{l}\text { Adjusted B } \\
\text { coefficient } \\
(95 \% \mathrm{CI})\end{array}$ & $\begin{array}{l}P \\
\text { value }\end{array}$ & $\begin{array}{l}\text { Crude B } \\
\text { coefficient } \\
(95 \% \mathrm{CI})\end{array}$ & $\begin{array}{l}P \\
\text { value }\end{array}$ & $\begin{array}{l}\text { Adjusted B } \\
\text { coefficient } \\
(95 \% \mathrm{CI})\end{array}$ & $\begin{array}{l}P \\
\text { value }\end{array}$ \\
\hline 3-4 drugs & $\begin{array}{l}-2.32(-10.76 \\
6.01)\end{array}$ & 0.58 & & & $\begin{array}{l}-4.22 \\
(-9.06 \\
0.54)\end{array}$ & 0.08 & & & $\begin{array}{l}-2.75 \\
(-7.67 \\
2.08)\end{array}$ & 0.27 & & \\
\hline$>4$ drugs & $\begin{array}{l}-2.74(-11.48 \\
5.80)\end{array}$ & 0.53 & & & $\begin{array}{l}-3.72 \\
(-8.74 \\
1.16)\end{array}$ & 0.14 & & & $\begin{array}{l}-0.12 \\
(-5.23 \\
4.83)\end{array}$ & 0.96 & & \\
\hline \multicolumn{13}{|l|}{ llbumin $(<35 \mathrm{~g} / \mathrm{l})$} \\
\hline $35-40 \mathrm{~g} / \mathrm{l}$ & $\begin{array}{l}8.36(0.65 \\
16.27)\end{array}$ & 0.03 & $\begin{array}{l}6.29 \\
(-0.39 \\
13.17)\end{array}$ & 0.09 & $\begin{array}{l}3.07 \\
(-1.47 \\
7.76)\end{array}$ & 0.19 & & & $\begin{array}{l}1.97 \\
(-2.65 \\
6.77)\end{array}$ & 0.41 & & \\
\hline$>40 \mathrm{~g} / \mathrm{l}$ & $\begin{array}{l}8.86(0.66, \\
17.27)\end{array}$ & 0.03 & $\begin{array}{l}2.47 \\
(-4.92 \\
10.01)\end{array}$ & 0.54 & $\begin{array}{l}3.76 \\
(-1.07 \\
8.75)\end{array}$ & 0.13 & & & $\begin{array}{l}2.36 \\
(-2.55 \\
7.46)\end{array}$ & 0.35 & & \\
\hline \multicolumn{13}{|c|}{ Hemoglobin $(<10 \mathrm{~g} / \mathrm{dl})$} \\
\hline $10-11 \mathrm{~g} / \mathrm{dl}$ & $\begin{array}{l}9.44(2.03 \\
16.82)\end{array}$ & 0.01 & $\begin{array}{l}7.27(0.70 \\
13.72)\end{array}$ & 0.04 & $\begin{array}{l}1.84 \\
(-2.59 \\
6.26)\end{array}$ & 0.41 & & & $\begin{array}{l}5.05(0.66 \\
9.40)\end{array}$ & 0.02 & & \\
\hline$>11 \mathrm{~g} / \mathrm{dl}$ & $\begin{array}{l}2.39(-4.75 \\
9.59)\end{array}$ & 0.51 & $\begin{array}{l}2.09 \\
(-3.94 \\
8.13)\end{array}$ & 0.53 & $\begin{array}{l}-0.59 \\
(-4.89 \\
3.71)\end{array}$ & 0.78 & & & $\begin{array}{l}2.65 \\
(-1.58 \\
6.91)\end{array}$ & 0.22 & & \\
\hline \multicolumn{13}{|l|}{ Vintage ( $\leq 12$ months) } \\
\hline 3-24 months & $\begin{array}{l}8.65(0.83 \\
16.80)\end{array}$ & 0.03 & $\begin{array}{l}10.47 \\
(3.57 \\
17.58)\end{array}$ & 0.008 & $\begin{array}{l}-0.44 \\
(-5.09 \\
4.40)\end{array}$ & 0.85 & & & $\begin{array}{l}7.48(3.04 \\
12.19)\end{array}$ & 0.001 & $\begin{array}{l}9.10 \\
(5.03 \\
13.46)\end{array}$ & $<0.001$ \\
\hline$>24$ months & $\begin{array}{l}5.70(-1.36 \\
13.03)\end{array}$ & 0.12 & $\begin{array}{l}9.71(3.33 \\
16.30)\end{array}$ & 0.007 & $\begin{array}{l}1.52 \\
(-2.68 \\
5.87)\end{array}$ & 0.48 & & & $\begin{array}{l}3.40(-0.6 \\
7.63)\end{array}$ & 0.107 & $\begin{array}{l}6.30 \\
(2.54 \\
10.30)\end{array}$ & 0.003 \\
\hline \multicolumn{13}{|c|}{ ID access (Fistula/graft) } \\
\hline $\begin{array}{l}\text { jemi-permanent } \\
\text { lialysis catheter }\end{array}$ & $\begin{array}{l}-8.44(-16.35 \\
-0.89)\end{array}$ & 0.03 & & & $\begin{array}{l}-6.20 \\
(-10.69 \\
-1.90)\end{array}$ & 0.006 & $\begin{array}{l}-6.11 \\
(-10.38 \\
-1.94)\end{array}$ & 0.008 & $\begin{array}{l}-3.88 \\
(-8.62 \\
0.57)\end{array}$ & 0.09 & & \\
\hline $\begin{array}{l}\text { Cemporary dialysis } \\
\text { satheter }\end{array}$ & $\begin{array}{l}-6.75(-14.71 \\
1.01)\end{array}$ & 0.09 & & & $\begin{array}{l}-5.59 \\
(-10.12 \\
-1.17)\end{array}$ & 0.01 & $\begin{array}{l}-5.30 \\
(-9.51 \\
-1.16)\end{array}$ & 0.02 & $\begin{array}{l}-3.00 \\
(-7.75 \\
1.59)\end{array}$ & 0.21 & & \\
\hline \multicolumn{13}{|c|}{ Number of comorbidities $(0) \$$} \\
\hline L & $\begin{array}{l}-11.40(-22.62 \\
-0.52)\end{array}$ & 0.04 & $\begin{array}{l}-15.31 \\
(-25.17 \\
-5.76)\end{array}$ & 0.005 & $\begin{array}{l}-0.12 \\
(-6.52 \\
6.08)\end{array}$ & 0.96 & $\begin{array}{l}-3.28 \\
(-9.11 \\
2.40)\end{array}$ & 0.29 & $\begin{array}{l}-10.04 \\
(-16.60 \\
-3.74)\end{array}$ & 0.003 & $\begin{array}{l}-9.55 \\
(15.58 \\
-3.82)\end{array}$ & 0.003 \\
\hline$?$ & $\begin{array}{l}-11.14(-22.79 \\
0.13)\end{array}$ & 0.06 & $\begin{array}{l}-13.20 \\
(-23.30 \\
-3.46)\end{array}$ & 0.01 & $\begin{array}{l}-4.55 \\
(-11.20 \\
1.88)\end{array}$ & 0.17 & $\begin{array}{l}-3.36 \\
(-9.64 \\
2.72)\end{array}$ & 0.31 & $\begin{array}{l}-6.51 \\
(-13.33 \\
0.021)\end{array}$ & 0.059 & $\begin{array}{l}-6.51 \\
(-12.96 \\
-0.57)\end{array}$ & 0.04 \\
\hline 3 & $\begin{array}{l}-21.49(35.22, \\
-8.03)\end{array}$ & 0.002 & $\begin{array}{l}-29.97 \\
(-41.47 \\
-18.32)\end{array}$ & $<0.001$ & $\begin{array}{l}-9.49 \\
(-17.33 \\
-1.81)\end{array}$ & 0.01 & $\begin{array}{l}-9.06 \\
(-16.42 \\
-1.84)\end{array}$ & 0.02 & $\begin{array}{l}-10.81 \\
(-18.84 \\
-3.012)\end{array}$ & 0.009 & $\begin{array}{l}-12.55 \\
(-19.97 \\
-5.60)\end{array}$ & 0.001 \\
\hline
\end{tabular}

*Adjusted for clustering of patients within hemodialysis centers.

\$Comorbidities include hypertension, heart failure, hepatitis B or C, cerebrovascular disease, HIV/AIDS and gout

Table 3. Factors associated with BKD, EKD and SPKD domains 
:nIndependent

les (reference) group)
Effects of kidney disease

coefficient value coefficient value

(95\% CI)

(95\% CI)

$7.17(-0.11, \quad 0.05$

14.32)

Male

Crude B P

coefficient value

(95\% CI)

$.95(-4.49$

$\begin{array}{llll}\text { Crude } B & P & \text { Adjusted } B & P\end{array}$

$\begin{array}{ll}\text { Adjusted B } & \mathrm{P} \\ \text { coefficient } & \text { value }\end{array}$

( $95 \%$ CI)

$6.84(-0.35, \quad 0.07 \quad 5.51$

14.03)
Symptom problem of kidney disease

\begin{tabular}{|c|c|c|c|}
\hline $\begin{array}{l}\text { Crude B } \\
\text { coefficient } \\
(95 \% \text { CI })\end{array}$ & $\begin{array}{l}P \\
\text { value }\end{array}$ & $\begin{array}{l}\text { Adjusted B } \\
\text { coefficient } \\
(95 \% \mathrm{CI})\end{array}$ & $\begin{array}{l}P \\
\text { value }\end{array}$ \\
\hline $\begin{array}{l}5.51 \\
-4.75 \\
5.66)\end{array}$ & 0.29 & $\begin{array}{l}11.98 \\
(2.82, \\
21.18)\end{array}$ & 0.01 \\
\hline $\begin{array}{l}7.30 \\
-4.46 \\
19.91)\end{array}$ & 0.23 & $\begin{array}{l}11.49 \\
(0.50 \\
23.35)\end{array}$ & 0.05 \\
\hline $\begin{array}{l}9.87 \\
-2.10 \\
2.39)\end{array}$ & 0.11 & $\begin{array}{l}13.611 \\
(1.42, \\
26.29)\end{array}$ & 0.04 \\
\hline
\end{tabular}

Age ( $<45$ years)

45-60 years

$\begin{array}{llll}-0.71(-9.23, & 0.87 & 1.16(-7.12, & 0.80\end{array}$

$2.25(-6.31$

10.82)

10.49)

$0.78 \quad 3.02(-5.98$

12.02)

$(-12.81$,

9.70)

$-1.70 \quad 0.78$

Education (Primary school and

less)

\begin{tabular}{|c|c|c|c|c|c|c|c|c|c|c|}
\hline Secondary school & $\begin{array}{l}0.11(-9.22, \\
9.02)\end{array}$ & 0.98 & & & $\begin{array}{l}0.51(-8.69 \\
9.44)\end{array}$ & 0.91 & & $\begin{array}{l}3.75 \\
(-9.30 \\
16.34)\end{array}$ & 0.87 & \\
\hline Post-secondary & $\begin{array}{l}5.68(-2.58 \\
13.77)\end{array}$ & 0.17 & & & $\begin{array}{l}7.51(-0.93 \\
15.87)\end{array}$ & 0.08 & & $\begin{array}{l}-0.94 \\
(-12.55 \\
10.45)\end{array}$ & 0.56 & \\
\hline \multicolumn{11}{|c|}{ Marital status (Never married) } \\
\hline $\begin{array}{l}\text { Married/living } \\
\text { together }\end{array}$ & $\begin{array}{l}0.17(-7.86, \\
8.32)\end{array}$ & 0.96 & & & $\begin{array}{l}0.04(-8.49 \\
8.62)\end{array}$ & 0.99 & & $\begin{array}{l}5.81 \\
(-5.53 \\
17.27)\end{array}$ & 0.32 & \\
\hline Separated/widowed & $\begin{array}{l}-8.12 \\
(-18.10 \\
2.02)\end{array}$ & 0.11 & & & $\begin{array}{l}1.52(-9.05 \\
12.18)\end{array}$ & 0.78 & & $\begin{array}{l}-0.87 \\
(-14.96 \\
13.41)\end{array}$ & 0.90 & \\
\hline \multicolumn{11}{|c|}{ Employment (Unemployed) } \\
\hline Employed & $\begin{array}{l}9.78(2.34 \\
17.31)\end{array}$ & 0.01 & $\begin{array}{l}10.29(3.20 \\
17.49)\end{array}$ & 0.009 & $\begin{array}{l}12.35(4.72, \\
19.98)\end{array}$ & 0.002 & & $\begin{array}{l}-0.12 \\
(10.91 \\
10.76)\end{array}$ & 0.98 & \\
\hline $\begin{array}{l}\text { Retired } \\
8.12(-1.92,18.43)\end{array}$ & & 0.12 & $\begin{array}{l}10.57(-0.96 \\
22.57)\end{array}$ & 0.10 & $\begin{array}{l}6.33(-3.93 \\
16.60)\end{array}$ & 0.23 & & $\begin{array}{l}4.01 \\
(-10.56, \\
18.91)\end{array}$ & 0.59 & \\
\hline \multicolumn{11}{|l|}{ Ubudehe category (1) } \\
\hline 2 & $\begin{array}{l}8.34(-5.78 \\
22.63)\end{array}$ & 0.25 & & & $\begin{array}{l}3.51(-11.22, \\
18.52)\end{array}$ & 0.64 & & $\begin{array}{l}8.71 \\
(-11.00 \\
28.62)\end{array}$ & 0.39 & \\
\hline 3 & $\begin{array}{l}7.09(-5.54 \\
20.15)\end{array}$ & 0.28 & & & $\begin{array}{l}3.06(-9.93 \\
16.67)\end{array}$ & 0.65 & & $\begin{array}{l}6.89 \\
(-10.77, \\
25.05)\end{array}$ & 0.45 & \\
\hline \multicolumn{11}{|c|}{ Health insurance coverage ( $<100 \%$ coverage) } \\
\hline $100 \%$ coverage & $\begin{array}{l}1.60(-5.85 \\
8.88)\end{array}$ & 0.66 & & & $\begin{array}{l}-2.18(-9.81 \\
5.42)\end{array}$ & 0.57 & & $\begin{array}{l}13.03 \\
(3.06 \\
22.81)\end{array}$ & 0.01 & $\begin{array}{l}9.57(0.01, \quad 0.05 \\
18.74)\end{array}$ \\
\hline \multicolumn{11}{|c|}{ Number of HD per week (Twice) } \\
\hline Thrice & $\begin{array}{l}-0.56(-9.74 \\
8.20)\end{array}$ & 0.90 & & & $\begin{array}{l}-11.83 \\
(-20.59 \\
-3.07)\end{array}$ & 0.009 & & $\begin{array}{l}2.21 \\
(-10.46, \\
14.46)\end{array}$ & 0.72 & \\
\hline \multicolumn{11}{|c|}{ Number of HD in the past 30 days $(\leq 10)$} \\
\hline$>10$ & $\begin{array}{l}-1.34(-8.75 \\
6.02)\end{array}$ & 0.72 & & & $\begin{array}{l}-9.08 \\
(-16.56 \\
-1.62)\end{array}$ & 0.01 & $\begin{array}{l}-7.70 \\
(-14.81 \\
-0.59)\end{array}$ & $\begin{array}{l}-1.91 \\
(-12.22, \\
8.34)\end{array}$ & 0.71 & \\
\hline \multicolumn{11}{|c|}{ Hospitalized in the last 6 months (No) } \\
\hline Yes & $\begin{array}{l}-3.73 \\
(-10.62, \\
3.11)\end{array}$ & 0.28 & & & $\begin{array}{l}-8.30 \\
(-15.42, \\
-1.15)\end{array}$ & 0.025 & & $\begin{array}{l}0.75 \\
(-9.08 \\
10.53)\end{array}$ & 0.87 & \\
\hline
\end{tabular}

Number of medications taking ( $<3$ drugs) 


\begin{tabular}{|c|c|c|c|c|c|c|c|c|c|c|c|c|}
\hline \multirow{2}{*}{$\begin{array}{l}\text { "nIndependent } \\
\text { les (reference) group) }\end{array}$} & \multicolumn{4}{|c|}{ Burden of kidney disease } & \multicolumn{4}{|c|}{ Effects of kidney disease } & \multicolumn{4}{|c|}{ Symptom problem of kidney disease } \\
\hline & $\begin{array}{l}\text { Crude B } \\
\text { coefficient } \\
(95 \% \mathrm{CI})\end{array}$ & $\begin{array}{l}P \\
\text { value }\end{array}$ & $\begin{array}{l}\text { Adjusted B } \\
\text { coefficient } \\
(95 \% \mathrm{CI})\end{array}$ & $\begin{array}{l}P \\
\text { value }\end{array}$ & $\begin{array}{l}\text { Crude B } \\
\text { coefficient } \\
(95 \% \mathrm{CI})\end{array}$ & $\begin{array}{l}P \\
\text { value }\end{array}$ & $\begin{array}{l}\text { Adjusted B } \\
\text { coefficient } \\
(95 \% \mathrm{CI})\end{array}$ & $\begin{array}{l}P \\
\text { value }\end{array}$ & $\begin{array}{l}\text { Crude B } \\
\text { coefficient } \\
(95 \% \text { CI })\end{array}$ & $\begin{array}{l}P \\
\text { value }\end{array}$ & $\begin{array}{l}\text { Adjusted B } \\
\text { coefficient } \\
(95 \% \mathrm{CI})\end{array}$ & $\begin{array}{l}P \\
\text { value }\end{array}$ \\
\hline 3-4 drugs & $\begin{array}{l}-5.10 \\
(-14.33 \\
3.96)\end{array}$ & 0.27 & & & $\begin{array}{l}-5.69 \\
(-15.26 \\
3.87)\end{array}$ & 0.25 & & & $\begin{array}{l}-7.48 \\
(-20.31 \\
5.16)\end{array}$ & 0.25 & & \\
\hline$>4$ drugs & $\begin{array}{l}1.64(-7.95 \\
10.92)\end{array}$ & 0.73 & & & $\begin{array}{l}-6.07 \\
(-15.87 \\
3.72)\end{array}$ & 0.23 & & & $\begin{array}{l}-12.43 \\
(-25.73 \\
0.52)\end{array}$ & 0.06 & & \\
\hline \multicolumn{13}{|l|}{ Albumin $(<35 \mathrm{~g} / \mathrm{l})$} \\
\hline $35-40 \mathrm{~g} / 1$ & $\begin{array}{l}7.24(-1.29 \\
16.14)\end{array}$ & 0.10 & & & $\begin{array}{l}2.19 \\
(-6.89,11.27)\end{array}$ & 0.64 & & & $\begin{array}{l}-1.02 \\
(-13.24 \\
11.61)\end{array}$ & 0.87 & & \\
\hline$>40 \mathrm{~g} / \mathrm{l}$ & $\begin{array}{l}9.62(0.51 \\
19.08)\end{array}$ & 0.04 & & & $\begin{array}{l}2.71(-7.01 \\
12.44)\end{array}$ & 0.58 & & & $\begin{array}{l}0.36 \\
(-12.65 \\
13.77)\end{array}$ & 0.95 & & \\
\hline \multicolumn{13}{|c|}{ Hemoglobin $(<10 \mathrm{~g} / \mathrm{dl})$} \\
\hline $10-11 \mathrm{~g} / \mathrm{dl}$ & $\begin{array}{l}9.03(0.74 \\
17.25)\end{array}$ & 0.03 & & & $\begin{array}{l}4.80(-3.93 \\
13.54)\end{array}$ & 0.28 & & & $\begin{array}{l}11.12 \\
(-0.50 \\
22.68)\end{array}$ & 0.06 & & \\
\hline$>11 \mathrm{~g} / \mathrm{dl}$ & $\begin{array}{l}1.17(-6.81 \\
9.21)\end{array}$ & 0.77 & & & $\begin{array}{l}2.84(-5.58 \\
11.29)\end{array}$ & 0.51 & & & $\begin{array}{l}6.05 \\
(-5.16 \\
17.34)\end{array}$ & 0.29 & & \\
\hline \multicolumn{13}{|c|}{ Vintage ( $\leq 12$ months) } \\
\hline 13-24 months & $\begin{array}{l}5.10(-3.66 \\
14.44)\end{array}$ & 0.26 & & & $\begin{array}{l}4.82(-4.16 \\
13.88)\end{array}$ & 0.30 & & & $\begin{array}{l}18.70 \\
(6.97 \\
31.03)\end{array}$ & 0.002 & $\begin{array}{l}16.28 \\
(5.20 \\
27.82)\end{array}$ & 0.008 \\
\hline$>24$ months & $\begin{array}{l}-0.01(-7.95 \\
8.35)\end{array}$ & 0.99 & & & $\begin{array}{l}-2.76 \\
(-10.89 \\
5.44)\end{array}$ & 0.51 & & & $\begin{array}{l}11.61 \\
(1.001 \\
22.68)\end{array}$ & 0.03 & $\begin{array}{l}9.74 \\
(-0.35 \\
20.31)\end{array}$ & 0.07 \\
\hline \multicolumn{13}{|c|}{ HD access (Fistula/graft) } \\
\hline $\begin{array}{l}\text { Semiparmanent } \\
\text { dialysis catheter }\end{array}$ & $\begin{array}{l}-8.59 \\
(-17.38 \\
-0.37)\end{array}$ & 0.04 & $\begin{array}{l}-8.01 \\
(-16.95 \\
0.51)\end{array}$ & 0.09 & $\begin{array}{l}-2.87 \\
(-11.30 \\
5.64)\end{array}$ & 0.50 & & & $\begin{array}{l}-8.89 \\
(-21.34 \\
2.92)\end{array}$ & 0.15 & & \\
\hline $\begin{array}{l}\text { Temporary dialysis } \\
\text { catheter }\end{array}$ & $\begin{array}{l}-10.78 \\
(-19.59 \\
-2.28)\end{array}$ & 0.01 & $\begin{array}{l}-10.71 \\
(-19.48 \\
-2.32)\end{array}$ & 0.02 & $\begin{array}{l}-1.14 \\
(-10.26 \\
7.97)\end{array}$ & 0.80 & & & $\begin{array}{l}-4.32 \\
(-16.83 \\
7.84)\end{array}$ & 0.49 & & \\
\hline \multicolumn{13}{|c|}{ Number of comorbidities $(0) \$$} \\
\hline 1 & $\begin{array}{l}-9.66 \\
(-22.58 \\
2.62)\end{array}$ & 0.13 & $\begin{array}{l}-17.32 \\
(-29.95 \\
-5.63)\end{array}$ & 0.009 & $\begin{array}{l}-6.32 \\
(-18.76 \\
6.12)\end{array}$ & 0.32 & $\begin{array}{l}-5.89 \\
(-18.13 \\
6.34)\end{array}$ & 0.36 & $\begin{array}{l}-22.96 \\
(-40.51 \\
-6.03)\end{array}$ & 0.01 & $\begin{array}{l}-19.93 \\
(-36.47 \\
-4.05)\end{array}$ & 0.02 \\
\hline 2 & $\begin{array}{l}-13.45 \\
(-26.87 \\
-0.69)\end{array}$ & 0.04 & $\begin{array}{l}-14.96 \\
(-28.29 \\
-2.676)\end{array}$ & 0.03 & $\begin{array}{l}-13.17 \\
(-26.14 \\
-0.18)\end{array}$ & 0.05 & $\begin{array}{l}-15.53 \\
(-27.99 \\
-3.06)\end{array}$ & 0.02 & $\begin{array}{l}-19.00 \\
(-37.23 \\
-1.43)\end{array}$ & 0.04 & $\begin{array}{l}-19.16 \\
(-36.56 \\
-2.71)\end{array}$ & 0.03 \\
\hline 3 & $\begin{array}{l}-14.78 \\
(-30.54 \\
0.46)\end{array}$ & 0.06 & $\begin{array}{l}-17.62 \\
(-33.01 \\
-2.97)\end{array}$ & 0.03 & $\begin{array}{l}-17.36 \\
(-33.09 \\
-1.62)\end{array}$ & 0.03 & $\begin{array}{l}-19.66 \\
(-34.92 \\
-4.41)\end{array}$ & 0.01 & $\begin{array}{l}-26.47 \\
(-47.96 \\
-5.51)\end{array}$ & 0.01 & $\begin{array}{l}-33.27 \\
(-53.11 \\
-14.20)\end{array}$ & 0.001 \\
\hline
\end{tabular}

*Adjusted for clustering of patients within hemodialysis centers. \$ Comorbidities include hypertension, heart failure, hepatitis B or C, cerebrovascular disease, HIV/AIDS and gout

Our results showed higher overall KDQOL score and all five domains scores in patients on in-centre maintenance hemodialysis at King Faisal Hospital and lower scores at CHUK-CHUB in-centre hemodialysis unit. This discrepancy might be related to patient's choice due to the fact that KFH is the most accredited hospital of the country, was the first to have HD unit in Kigali with more HD beds than other centers, specialized medical staff, sufficient human resources and materials. In addition, the national referral board office that transfers patients for kidney transplantation is located at KFH and there is a possibility to access HD using 
health insurance coverage as in other in-centre dialysis units. The majority of patients managed at CHUK and CHUB are referred from rural areas with lesser socioeconomic status making their dialysis units less frequently used for chronic maintenance HD.

Our findings showed positive correlation between dialysis vintage and higher QoL scores, likely reflecting the cognitive adaptation of patients dialyzed for a long period. (40)(25)(21)(38)(32) In this study, the number of comorbidities was in direct proportion with worse quality of life score, affecting all HRQOL domains. Jieun Cha et al (23) in Korea demonstrated a significant poor HRQOL associated with the high number of comorbidities $(p<0.001)$.

Study limitations: There are a number of limitations that need to be acknowledged. First, while KDQOL-36 $6^{\mathrm{TM}}$ instrument has been validated in other contexts, it is yet to be validated in Rwandan context and there is no validated translation form of KDQOL-36 ${ }^{\mathrm{TM}}$ survey into the local language (Kinyarwanda) before data collection. Therefore, there might have been some instance of misinterpretations when translating the questionnaire during data collection. Second, being a cross-sectional study makes it difficult to infer causality including the determination of causes of low HRQOL found. Third, while the sample size appears relatively small, we included nearly all eligible patients across the in-centre hemodialysis centres. Fourth, we did not include community dialysis units - there were three community dialysis unities in the country during the study period. Lastly, our analysis did not thoroughly explore some metrics that clinicians/patients frequently report as barriers to care and to QOL such as financial constraints (out of pocket costs or yearly income) and travel to HD units.

\section{Conclusion}

Patients on in-centre hemodialysis in Rwanda have a significantly low HRQOL scores. The lowest score was found on burden of kidney disease and physical composite summary domains. There is a notable difference of HRQOL scores between hemodialysis units. Factors associated with overall HRQOL found were sex, employment status, number of comorbidities, dialysis vintage, and hemoglobin level. Optimizing medical and biomedical management of dialysis patients and finding ways to make dialysis less obstructive to maintain employment should improve their HRQOL. The majority of patients on hemodialysis have higher socioeconomic status reflecting the financial constraints and the social impact to access and maintain renal replacement therapy in resource limited settings. As such, improving equitable access to RRT should remain a priority.

We recommend local validation of the KDQOL survey instrument, use of different validated poverty index to further evaluate the impact of poverty on quality of life in Rwandan context to ensure outcomes of the survey and help clinical decision making routinely. Further studies on HD adequacy to both in-centre as well as community based HD units, and comparison between using internationally accepted measures of dialysis adequacy $(\mathrm{Kt} / \mathrm{V})$ and QOL measures which are cheap and easy to administer in low income countries are also recommended.

\section{Table 4. Health related quality of life of patients treated with hemodialysis from different studies using the KDQOL-36 ${ }^{\mathrm{TM}}$}

\begin{tabular}{llllll}
\hline \multicolumn{3}{c}{ Country } & & & \\
\hline & Rwanda ${ }^{*}$ & Kenya(24) & Saudi Arabia(65) & USA(64) & USA(46) \\
\hline PCS & 37.33 & 39.09 & 37.4 & 38 & 36.6 \\
MCS & 44.74 & 41.87 & 43.5 & 51.8 & 49.0 \\
\hline BKD & 20.01 & 16.15 & 31.5 & 53.2 & 51.3 \\
EKD & 53.48 & 67.63 & 56.5 & 76.6 & 78.1 \\
\hline SPKD & 58.22 & 73.46 & 74 & 80.7 & 73.0 \\
\hline
\end{tabular}

PCS physical component summary, MCS mental component summary, BKD burden of kidney disease component summary, EKD effect of kidney disease component summary, SPKD symptoms and problem of kidney disease component summary USA United States of America \#present study

\section{Declarations}

Page $12 / 16$ 
- Ethics approval and consent to participate: All methods were carried out in accordance with relevant guidelines and regulations. The approval to carry out this study was given by the University of Rwanda Institutional Review Board (IRB) No 053/CMHS IRB/2020 as well as the ethics committee of University Teaching Hospital of Kigali, referenced as Ref:

EC/CHUK/102/2020, Rwanda military hospital, referenced as Ref: 046/RMH/COMDT/2021, University Teaching Hospital of Butare, referenced as Ref: CHUB/DG/SA/12/2734/2020 and King Faisal Hospital.

All study participants signed written informed consent prior to study enrollment.

- Consent for publication: Not applicable. No individual personal data were included in the study. All patients provided the necessary consent to participate in the present study.

- Availability of data and materials:The datasets generated and analyzed during the current study are not publicly available because we are not allowed to share individual level data. However additional information about the data is available from the corresponding author on reasonable request.

- Competing interests: All the authors have declared no conflicts of interest

- Funding: The study was not funded

- Authors' contributions:

A. Gloria SHUMBUSHO conceived, designed and coordinated the study, collected data and wrote the manuscript.

B. Jules KABAHIZI participated in the design of the study and critically reviewed the manuscript.

C. Marla MCKNIGHT participated in the design of the study and critically reviewed the manuscript.

D. Celestin HATEGEKA contributed to the study design, performed statistical analysis and critically reviewed the manuscript.

E. Marianne Vidler reviewed the manuscript.

All authors read and approved the final manuscript.

- Acknowledgements: We would like to thank the nursing staff in the dialysis center and the patients who participated in the study. Celestin Hategeka received support through a Banting Postdoctoral Fellowship from the Canadian Institutes of Health Research

\section{References}

1. White SL, Chadban SJ, Jan S, Chapman JR, Cass A. How can we achieve global equity in provision of renal replacement therapy? Bull World Health Organ. 2008;86(3):229-37.

2. Atlas IDFD. International Diabetes Federation. Vol. 266, The Lancet. 1955. 134-137 p.

3. Metrics GH. Chronic kidney disease - Level 3 cause. 2020;396:152-3.

4. Coates MM, Kintu A, Gupta N, Wroe EB, Adler AJ, Kwan GF, et al. Burden of non-communicable diseases from infectious causes in 2017: a modelling study. Lancet Glob Heal. 2020;8(12):e1489-98.

5. Joshi R, John O, Jha V. The Potential Impact of Public Health Interventions in Preventing Kidney Disease. Semin Nephrol [Internet]. 2017;37(3):234-44. Available from: http://dx.doi.org/10.1016/j.semnephrol.2017.02.004

6. Stanifer JW, Jing B, Tolan S, Helmke N, Mukerjee R, Naicker S, et al. The epidemiology of chronic kidney disease in subSaharan Africa: A systematic review and meta-analysis. Lancet Glob Heal. 2014;2(3):e174-81.

7. Liyanage T, Ninomiya T, Jha V, Neal B, Patrice HM, Okpechi I, et al. Worldwide access to treatment for end-stage kidney disease: A systematic review. Lancet. 2015;385(9981):1975-82.

8. Ashuntantang G, Osafo C, Olowu WA, Arogundade F, Niang A, Porter J, et al. Outcomes in adults and children with end-stage kidney disease requiring dialysis in sub-Saharan Africa: a systematic review. Lancet Glob Heal [Internet]. 2017;5(4):e408-17. Available from: http://dx.doi.org/10.1016/S2214-109X(17)30057-8 
9. Halle MP, Ashuntantang G, Kaze FF, Takongue C, Kengne AP. Fatal outcomes among patients on maintenance haemodialysis in sub-Saharan Africa: a 10-year audit from the Douala General Hospital in Cameroon. BMC Nephrol [Internet]. 2016;17(1):1-9. Available from: http://dx.doi.org/10.1186/s12882-016-0377-5

10. Rwanda Demographics 2020 (Population, Age, Sex, Trends) - Worldometer [Internet]. [cited 2021 Jul 2]. Available from: https://www.worldometers.info/demographics/rwanda-demographics/\#pop

11. Rwanda - Gross domestic product (GDP) per capita 1986-2026 | Statista [Internet]. [cited 2021 Jul 2]. Available from: https://www.statista.com/statistics/452130/gross-domestic-product-gdp-per-capita-in-rwanda/

12. Ezeanya C. Home-grown and grassroots-based strategies for determining inequality towards policy action: Rwanda's Ubudehe approach in perspective. World Inst Dev Econ Res. 2015;(January):17.

13. LODA: The pilot phase of Ubudehe categorization process kicks off countrywide [Internet]. [cited 2021 Jul 2]. Available from: https://www.loda.gov.rw/fr/newsmailer/news-singleview/news/the-pilot-phase-of-ubudehe-categorization-process-kicks-offcountrywide/?

tx_news_pi1\%5Bcontroller\%5D=News\&tx_news_pi1\%5Baction\%5D=detail\&cHash=e77862ec9f965382c3d726c777ef6c9e

14. USAID. Health insurance profile: Rwanda. African Strateg Heal. 2013;8-11.

15. National Strategy and Costed Action Plan for the Prevention and Control of Non-Communicable Diseases in Rwanda. 2020; (June).

16. Chironda G, Ngendahayo F, Mudasumbwa G, Dushimiyimana V, Jeanne Tuyisenge M, Kayitesi J, et al. Renal replacement therapy (RRT) in Rwanda: Benefits, challenges and recommendations. Vol. 76, Rwanda Medical Journal. 2019. p. 1-6.

17. Unlocking Rwanda's Potential to Reap the Demographic dividend. Published 2017. Accessed July2, 2021. http://www.statistics.gov.rw/file/6152/download?token=cttW07Pw. 2017.

18. Igiraneza G, Ndayishimiye B, Nkeshimana M, Dusabejambo V, Ogbuagu O. Clinical Profile and Outcome of Patients with Acute Kidney Injury Requiring Hemodialysis: Two Years' Experience at a Tertiary Hospital in Rwanda. Biomed Res Int. 2018;2018.

19. Bitunguhari L, Shahidi Twahirwa T, Niyomurengezi A, Mukiza J. The outcome of patients on hemodialysis at university teaching hospital of kigali (CHUK) in a period of september 2014 to march 2017 - A retrospective study on patients with acute and chronic renal failure. Rwanda Med J. 2019;76(3):1-7.

20. Chan CT, Blankestijn PJ, Dember LM, Gallieni M, Harris DCH, Lok CE, et al. Dialysis initiation, modality choice, access, and prescription: conclusions from a Kidney Disease: Improving Global Outcomes (KDIGO) Controversies Conference. Kidney Int. 2019;96(1):37-47.

21. Sesso R, Rodrigues-Neto JF, Ferraz MB. Impact of socioeconomic status on the quality of life of ESRD patients. Am J Kidney Dis. 2003;41(1):186-95.

22. Perl J, Karaboyas A, Morgenstern H, Sen A, Rayner HC, Vanholder RC, et al. Association between changes in quality of life and mortality in hemodialysis patients: Results from the DOPPS. Nephrol Dial Transplant. 2017;32(3):521-7.

23. Cha J, Han D. Health-related quality of life based on comorbidities among patients with end-stage renal disease. Osong Public Heal Res Perspect. 2020;11(4):194-200.

24. Domenick Sridharan N, Fish L, Yu L, Weisbord S, Jhamb M, Makaroun MS, et al. The associations of hemodialysis access type and access satisfaction with health-related quality of life. J Vasc Surg [Internet]. 2018;67(1):229-35. Available from: http://dx.doi.org/10.1016/j.jvs.2017.05.131

25. Yang F, Griva K, Lau T, Vathsala A, Lee E, Ng HJ, et al. Health-related quality of life of Asian patients with end-stage renal disease (ESRD) in Singapore. Qual Life Res. 2015;24(9):2163-71.

26. Dąbrowska-Bender M, Dykowska G, Żuk W, Milewska M, Staniszewska A. The impact on quality of life of dialysis patients with renal insufficiency. Patient Prefer Adherence. 2018;12:577-83.

27. Finkelstein FO, Wuerth D, Finkelstein SH. Health related quality of life and the CKD patient: Challenges for the nephrology community. Kidney Int [Internet]. 2009;76(9):946-52. Available from: http://dx.doi.org/10.1038/ki.2009.307

28. ESRD Quality Incentive Program I CMS [Internet]. [cited 2021 Jun 17]. Available from: https://www.cms.gov/Medicare/Quality-Initiatives-Patient-Assessment-Instruments/ESRDQIP 
29. Kidney Disease Quality of Life Instrument (KDQOL) I RAND [Internet]. [cited 2021 Apr 22]. Available from: https://www.rand.org/health-care/surveys_tools/kdqol.html

30. Hays R, Kallich JD, Mapes DL, Coons SJ, Amin N, Carter WB, et al. Kidney Disease Quality of Life Short Form (KDQOL-SF) version 1.3: A Manual for Use and Scoring. [Internet]. Rand. 1997. p. 1-39. Available from: https://www.rand.org/content/dam/rand/www/external/health/surveys_tools/kdqol/kdqol13man.pdf

31. Peipert JD, Nair D, Klicko K, Schatell DR, Hays RD. Kidney disease quality of life 36-item short form survey (KDQOL-36) normative values for the United States dialysis population and new single summary score. J Am Soc Nephrol. 2019;30(4):654-63.

32. Masina T, Chimera B, Kamponda M, Dreyer G. Health related quality of life in patients with end stage kidney disease treated with haemodialysis in Malawi: A cross sectional study. BMC Nephrol [Internet]. 2016;17(1):4-11. Available from: http://dx.doi.org/10.1186/s12882-016-0292-9

33. Kamau E, Kayima J, Otieno C, Maritim MC, Wanzala P. Health related quality of life of patients on maintenance haemodialysis at kenyatta national hospital. East Afr Med J. 2012;89(3):75-81.

34. Stephen Z. Fadem MD. Your Health and Well-Being Kidney Disease and Quality of Life (KDQOLTM-36) [Internet]. [cited 2021 Jul 22]. Available from: http://touchcalc.com/calculators/sf_36

35. Bagasha P, Namukwaya E, Leng M, Kalyesubula R, Mutebi E, Naitala R, et al. Comparison of the health-related quality of life of end stage kidney disease patients on hemodialysis and non-hemodialysis management in Uganda. BMC Palliat Care. 2021;20(1):1-13.

36. Robinson BM, Akizawa T, Jager KJ, Kerr PG, Saran R, Pisoni RL. Dialysis 3 Factors aff ecting outcomes in patients reaching end-stage kidney disease worldwide: diff erences in access to renal replacement therapy, modality use, and haemodialysis practices. www.thelancet.com [Internet]. 2016 [cited 2021 Apr 25];388. Available from:

http://dx.doi.org/10.1016/

37. Murali R, Sathyanarayana D, Muthusethupathy M. Assessment of quality of life in chronic kidney disease patients using the kidney disease quality of life-short formtm questionnaire in indian population: A community based study. Asian J Pharm Clin Res. 2015;8(1):271-4.

38. Zouari L, Omri S, Turki S, Maâlej M, Charfı N, Ben Thabet J, et al. Quality of life in chronic hemodialysis patients:about 71 cases. Tunis Med [Internet]. 2016;94(1):40-5. Available from: http://www.ncbi.nlm.nih.gov/pubmed/27525604

39. Mollaoğlu M. Quality of Life in Patients Undergoing Hemodialysis. 2013;

40. Alqahtani NA, Al-Metrek MA, Al-Alsheikh K, Elnazer WH. Quality of life among patients with chronic renal failure on hemodialysis at the military hospital in southern region of Saudi Arabia. MOJ Anat Physiol. 2019;6(5):6-9.

41. Eneanya ND, Maddux DW, Reviriego-mendoza MM, Larkin JW, Usvyat LA, Sande FM Van Der, et al. Longitudinal patterns of health-related quality of life and dialysis modality: a national cohort study. 2019;1-9.

42. Cohen DE, Lee A, Sibbel S, Benner D, Brunelli SM, Tentori F. Correction to: Use of the KDQOL-36 ${ }^{\text {TM }}$ for assessment of healthrelated quality of life among dialysis patients in the United States (BMC Nephrology (2019) 20:1 (112) DOI:

10.1186/s12882-019-1295-0). BMC Nephrol. 2019;20(1):1-9.

\section{Figures}


96 patients were dialyzed for ESKD

between September 2020 and March 2021:

King Faisal hospital: 45

Rwanda military hospital: 35

Kigali university teaching hospital: 9

Butare university teaching hospital: 7

No consent: 3

Aged less than 18 years : 2

Too sick to be recruited: 2

89 patients met eligibility criteria

ESKD: End stage kidney disease

Figure 1. Patients selection flow

Figure 1

Patients' selection flow

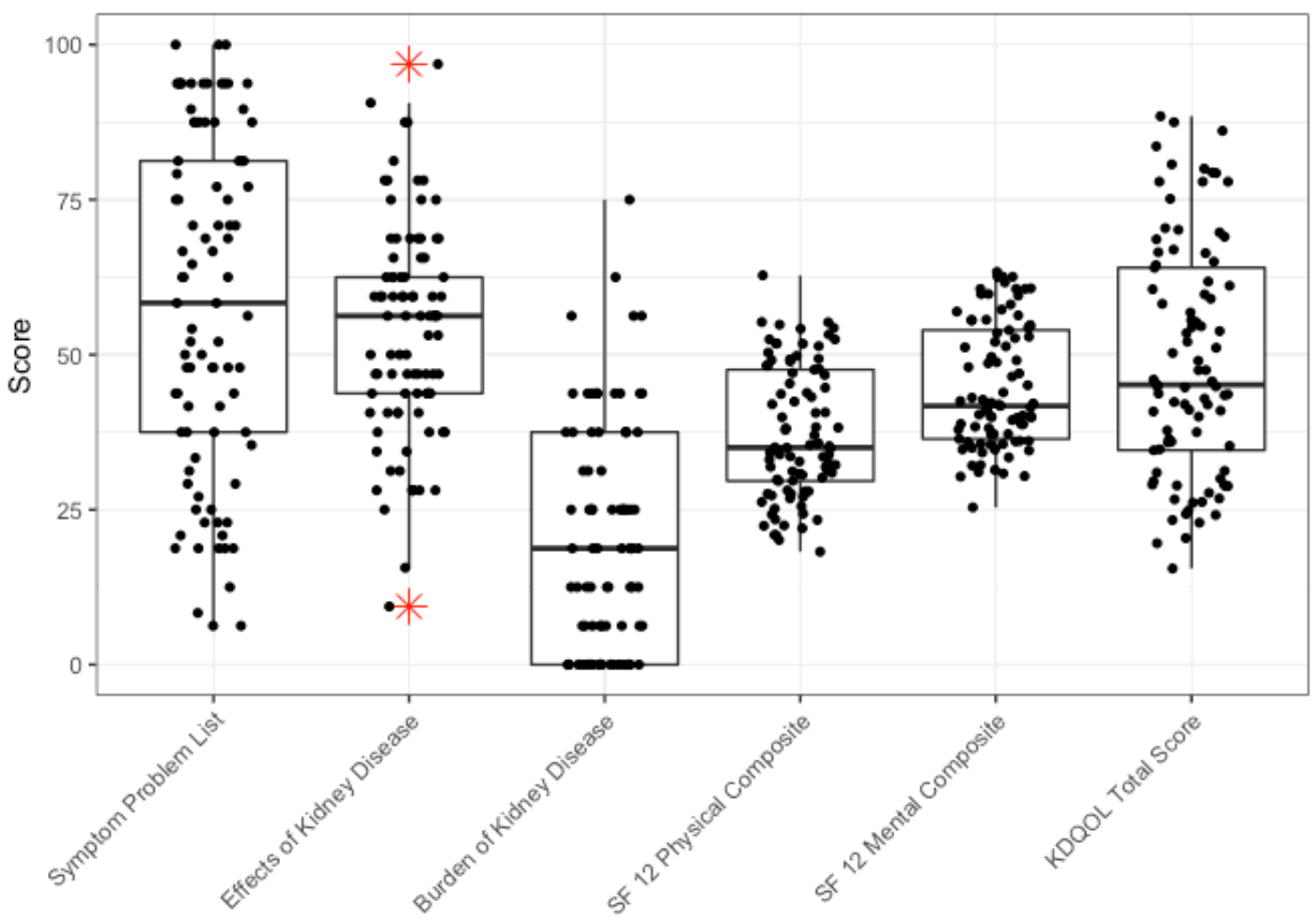

Figure 2

Distribution of HRQOL patients scores by KDQOL-36 ${ }^{\mathrm{TM}}$ domains 